\section{General Medicine and Surgery for Dental Practitioners}

Authors: Mark Greenwood, John G. Meechan

Publisher: Springer Nature, Switzerland

Language: English

ISBN: 978-3-319-97737-9

Edition: $3 / \mathrm{e}$

Publish Year: 2019

Pages: 253, illustrated

Price: $€ 96,29$

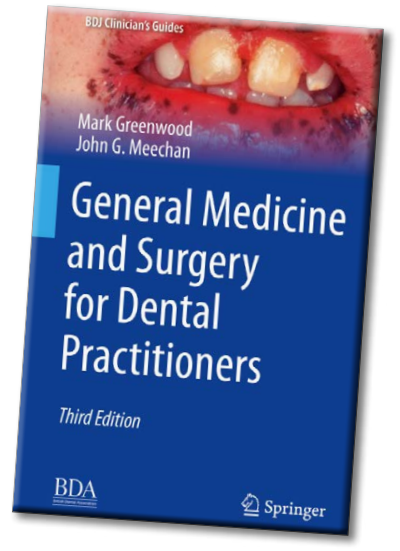

Iulia Ciolachi

DMD

Holistic Dental \& Medical Institute

of Bucharest-ROPOSTURO

Bucharest, Romania

e-mail:

driuliaciolachi@yahoo.ro

The third edition of General Medicine and Surgery for Dental Practitioners by Mark Greenwood and John G. Meechan provides a detailed overview of the medical and surgical conditions that affect patients seen in daily dental practice. The book has twenty chapters and ends with an index..

The first chapter focuses on the holistic patient assessment and main components of a medical history. The next nine chapters address the nine types of body systems and their conditions. They explain how to clinically recognize some general conditions, which is the protocol of dental treatment in certain conditions, in which cases the dental treatment can be done in the dental offices and when to be treated in hospital. The most common are cardiovascular, respiratory and gastrointestinal system, neurological disorders, liver disease, endocrine system, renal disorders, musculoskeletal system, hematology and patients with bleeding problems.

The following two chapters specifically talk about the pediatric patient and the older patient management in daily dental practice. The keys to successful treatment with this type of patient are: accurate medical history, good examination, rigorous preventive programs, dental intervention at times appropriate to medical care and regular follow-up.

Metabolic disorders, skin disorders, psychiatry, cancer, radiotherapy and chemotherapy and dental practice are the topics of the next four chapters.

Knowledge of all of these disorders will highlight patients who need special precautions to facilitate safe treatment. It is important that dental practitioners are aware of the most common disorders and their potential implications as they are likely to encounter them in clinical practice.

The next chapter presents the currently recommended contents of the emergency drug box as well as the equipment and basic management principles.

The following chapter discusses treatment for specific emergencies, teaches us how to recognize signs and symptoms of relevant medical emergencies and what medicines to administer. The subsequent chapter presents and underlines principles behind maintaining a safe working environment. The last chapter provides an overview of clinical immunology and how it interfaces with dentistry.

General Medicine and Surgery for Dental Practitioners is a book useful to all practitioners, helping them to better recognize and treat patients with certain conditions that can lead to true complications in combination with dental treatments. It is written in an easy way and is accompanied by numerous images to facilitate understanding.

d) http://www.stomaeduj.com 10.25241/stomaeduj.2020.7(2).bookreview.6

The Books Review is drafted in the reviewer's sole wording and illustrates his opinions. 